Revista Brasileira de Farmacognosia Brazilian Journal of Pharmacognosy 22(4): 714-723, Jul./Aug. 2012

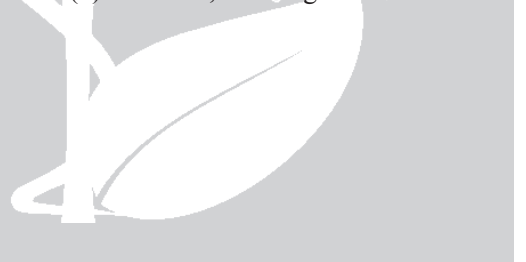

Article

Received 14 Oct 2011

Accepted 6 Nov 2011

Available online 15 May 2012

Keywords:

Acyclovir-resistant HSV

marine algae

marine natural products

secondary metabolites

ISSN 0102-695X

http://dx.doi.org/10.1590/S0102-

695X2012005000061

\section{Antiviral activity of extracts from Brazilian seaweeds against herpes simplex virus}

\author{
Angélica Ribeiro Soares, ${ }^{*}, 1$ Marcela C. S. Robaina, ${ }^{2}$ Gabriella \\ S. Mendes, ${ }^{2}$ Thalia S. L. Silva, ${ }^{1,3}$ Lísia M. S. Gestinari, ${ }^{1}$ Odinéia \\ S. Pamplona, ${ }^{1}$ Yocie Yoneshigue-Valentin, ${ }^{4}$ Carlos R. Kaiser, ${ }^{3}$ \\ Maria Teresa Villela Romanos ${ }^{2}$
}

\author{
${ }^{I}$ Núcleo em Ecologia e Desenvolvimento Sócio-Ambiental de Macaé, \\ Universidade Federal do Rio de Janeiro, Brazil, \\ ${ }^{2}$ Departamento de Virologia do Instituto de Microbiologia Prof. Paulo de \\ Góes, Cenctro de Ciências da Saúde, Universidade Federal do Rio de Janeiro, \\ Brazil, \\ ${ }^{3}$ Instituto de Química, Universidade Federal do Rio de Janeiro, Brazil, \\ ${ }^{4}$ Departamento de Botânica, Instituto de Biologia, Universidade Federal do Rio \\ de Janeiro, Brazil.
}

\begin{abstract}
Organic extracts of 36 species of marine algae (sixteen species of Rhodophyta, eight species of Ochrophyta and twelve species of Chlorophyta) from seven locations on the Brazilian coast were evaluated for their anti-HSV-1 and anti-HSV-2 activity resistant to Acyclovir (ACV). Activity tests in crude extracts, followed by the identification of the major compounds present, were performed for all species. The chemical profiles of all crude extracts were obtained by ${ }^{1} \mathrm{H}-\mathrm{NMR}$ and ${ }^{13} \mathrm{C}$-NMR spectroscopy. The percentage of extracts with antiviral activity was higher for HSV-1 (86.1\%) than for HSV-2 (55.5\%). The green algae Ulva fasciata and Codium decorticatum both showed the highest activity (99.9\%) against HSV-1, with triacylglycerols and fatty acids as the major components. The red alga Laurencia dendroidea showed good activity against HSV-1 (97.5\%) and the halogenated sesquiterpenes obtusol and (-)-elatol were identified as the major components in the extract. Against HSV-2, the green alga Penicillus capitatus (Chlorophyta) and Stypopodium zonale (Ochrophyta) were the most active (96.0 and 95.8\%). Atomaric acid, a meroditerpene, was identified as the major secondary metabolite in the $S$. zonale extract. These results reinforce the role of seaweeds as important sources of compounds with the potential to enter into the pipeline for development of new drugs against herpes simplex.
\end{abstract}

\section{Introduction}

Seaweeds provide a rich source of structurally diverse secondary metabolites. These are mainly terpenes, acetogenins and polyphenols, including many halogenated compounds (Maschek \& Baker, 2008). These secondary metabolites provide defense against herbivores (Pereira et al., 2004b; Lima et al., 2008), fouling organisms (Da Gama et al., 2008) and pathogens (Paul \& Ritson-Williams, 2008); they also play a role in reproduction (Amsler \& Fairhead, 2005), protection from UV radiation (Gomez et al., 1998) and as allelopathic agents (Beach et al., 2003). These compounds have shown some interesting pharmacological activities such as: antitumoral (Barbier et al., 2001), antiparasitic (Davyt et al., 2001), antibacterial (Vairappan, 2003), antiviral (Santos et al., 1999; Pereira et al., 2004a;
Soares et al., 2007), antioxidant (Nahas et al., 2007), and antifungal activity (de Oliveira et al., 2008). In particular, antiviral effects of sulfated polysaccharides and terpenes against a variety of enveloped viruses, such as Herpes Simplex Virus type 1 (HSV-1) and 2 (HSV-2), Human Immunodeficiency Virus (HIV), human cytomegalovirus, dengue viruses, respiratory syncytial and influenza viruses have been reported (Laillea et al., 1998; Ghosh et al., 2004; Cirne-Santos et al., 2008; Hidari et al., 2008).

At present, the availability of safe and potent antiviral agents against herpes viruses is far from ideal. Acyclovir (ACV) is the compound chosen for clinical use against HSV-1 and HSV-2 in systemic or topical therapy (Brown et al., 2002). Other ACV-related nucleoside analogs, all targeted against viral DNA synthesis, have recently been approved for human use (De Clercq, 2005). Although these compounds are potent and contribute to 
the overall reduction of morbidity associated with viral infection, the emergence of viral resistant variants after prolonged treatment in immunocompromised patients still occurs, which justifies the continuous search for novel antiherpetic agents (Jerome, 2005).

In this context, metabolites from algae represent interesting types of compounds to assay as promising antiviral agents. This study presents the in vitro antiherpetic properties and the chemical profiles of most active crude extracts of seaweeds from the Brazilian coast.

\section{Materials and Methods}

\section{Plant material}

Thirty-six species of macroalgae, belonging to three algal divisions (Rhodophyta, Ochrophyta and Chlorophyta), were collected from six sites in Rio de Janeiro state, on the southeastern Brazilian coast: Forno beach $\left(22^{\circ} 44^{\prime} 31.70^{\prime \prime} \mathrm{S}, 4^{\circ} 52^{\prime} 35.97^{\prime \prime O}\right)$, Rasa beach $\left(22^{\circ} 44^{\prime} 3.15^{\prime \prime} \mathrm{S}, 41^{\circ} 57^{\prime} 30.15^{\prime \prime O}\right)$, Francês Island $\left(22^{\circ} 24^{\prime} 6.46^{\prime \prime S}, 41^{\circ} 41^{\prime} 37.16^{\prime \prime O}\right)$, Tatagiba beach (2123'31.11"S, 4059'9.64"O), Cavaleiros beach $\left(22^{\circ} 24^{\prime} 18.50^{\prime \prime} \mathrm{S}, 41^{\circ} 47^{\prime} 42.48^{\prime \prime} \mathrm{O}\right)$ and Cabo Frio Island $\left(23^{\circ} 0^{\prime} 10.02^{\prime \prime S}, 420^{\prime} 24.43^{\prime \prime} \mathrm{O}\right)$, between February,
2006, and March, 2007. Five species of Chlorophyta were collected in Bahia state, on the northeast coast (176'38.86"S, 39¹0'54.95" W), in March, 2009 (Table 1). All algae were collected by A. R. Soares and identified by L. M. S. Gestinari and Y. Yoneshigue-Valentin. The algae were washed in seawater to eliminate associated organisms and air-dried. Voucher specimens were deposited at RFA (Thiers, 2008).

\section{Chemical analysis}

The air-dried algal material was powdered and extracted three times with dichloromethane:methanol (1:1) at room temperature, except the material collected in Bahia state, which was extracted three times with dichloromethane. After the evaporation of the solvent, all the crude extracts were analyzed by ${ }^{1} \mathrm{H}-\mathrm{NMR}$ (Nuclear Magnetic Resonance) (300 $\left.\mathrm{MHz}, \mathrm{CDCl}_{3}\right)$ and ${ }^{13} \mathrm{C}$ NMR (75 MHz, $\mathrm{CDCl}_{3}$ ) spectroscopy (Bruker Avance spectrometer using tetramethylsilane (TMS) as internal standard) and thin layer chromatography (Silica gel $\mathrm{GF}_{254}$ TLC plates, Merck) with $2 \% \mathrm{Ce}\left(\mathrm{SO}_{4}\right)_{2}$ in sulphuric acid as the spray detection reagent and heating the TLC plates at $100^{\circ} \mathrm{C}$. The crude extracts were used to perform antiherpetic activity evaluation.

Table 1. The checklist of the benthic marine macroalgae collected in this study.

\begin{tabular}{|c|c|c|c|c|}
\hline Phyllum & Sampling Site Habitat & Species & Order & Family \\
\hline \multirow{14}{*}{ 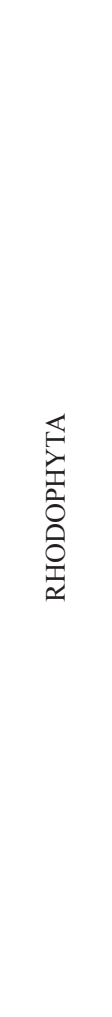 } & $\begin{array}{c}\text { Cabo Frio Island } \\
\left(23^{\circ} 0^{\prime} 10.02^{\prime \prime S}, 4200^{\prime} 24.43^{\prime \prime} \mathrm{O}\right) \\
\text { Intertidal }\end{array}$ & Laurencia dendroidea J. Agardh & Ceramiales & Rhodomelaceae \\
\hline & $\begin{array}{c}\text { Cavaleiros Beach } \\
\left(22^{\circ} 24^{\prime} 18.50^{\prime \prime} \mathrm{S}, 41^{\circ} 47^{\prime} 42.48^{\prime \prime O}\right) \\
\text { Intertidal }\end{array}$ & Corallina panizzoii Schnetter \& U. Richt. & Corallinales & Corallinaceae \\
\hline & & Jania crassa J.V. Lamour. & Corallinales & Corallinaceae \\
\hline & & $\begin{array}{c}\text { Centroceras clavulatum (C. Agardh in Kunth) Mont. in } \\
\text { Durieu de Maisonneuve }\end{array}$ & Ceramiales & Ceramiaceae \\
\hline & $\begin{array}{c}\text { Forno Beach } \\
\left(22^{\circ} 44^{\prime} 31.70^{\prime \prime} \mathrm{S}, 41^{\circ} 52^{\prime} 35.97^{\prime \prime O}\right)\end{array}$ & $\begin{array}{l}\text { Pterocladiella capillacea (S.G. Gmel.) Santel. \& } \\
\text { Hommers. }\end{array}$ & Gelidiales & Gelidiaceae \\
\hline & & Hypnea musciformis & Gigartinales & \\
\hline & & Laurencia dendroidea J. Agardh & Ceramiales & \\
\hline & $\begin{array}{c}\text { Francês Island } \\
\left(22^{\circ} 24^{\prime} 6.46^{\prime \prime} \mathrm{S}, 41^{\circ} 41^{\prime} 37.16^{\prime \prime} \mathrm{O}\right) \\
\text { Intertidal }\end{array}$ & Jania adhaerens J.V. Lamour. & Corallinales & Corallinaceae \\
\hline & \multirow{6}{*}{$\begin{array}{c}\text { Rasa Beach } \\
\left(22^{\circ} 44^{\prime} 3.15^{\prime \prime} \mathrm{S}, 41^{\circ} 57^{\prime} 30.15^{\prime \prime} \mathrm{O}\right) \\
\text { Intertidal }\end{array}$} & Hypnea spinella (C. Agardh) Kütz & Gigartinales & Cystocloniacae \\
\hline & & Spyrdia clavata Kütz & Ceramiales & Ceramiaceae \\
\hline & & Acantophora spicifera & & \\
\hline & & Bostrychia radicans (Mont.) Mont. in Orbigny & Ceramiales & Rhodomelaceae \\
\hline & & Cryptonemia seminervis (C. Agardh) J. Agardh & Halymeniales & Halymeniaceae \\
\hline & & Gracilaria domingensis (Kütz.) Sond. Ex Dickie & Gracilariales & Gracilariaceae \\
\hline
\end{tabular}




\begin{tabular}{|c|c|c|c|c|}
\hline \multicolumn{5}{|c|}{ Hypnea musciformis } \\
\hline & & $\begin{array}{c}\text { Plocamium brasiliense (Grev. in J.St.-Hil.) M. Howe \& } \\
\text { W.R.Taylor }\end{array}$ & Plocamiales & Plocamiaceae \\
\hline & & Osmundaria obtusiloba (C. Agardh) R. E. Norris & Ceramiales & Rhodomelaceae \\
\hline & & $\begin{array}{l}\text { Tricleocarpa cylindrica (J. Ellis \& Sol.) Huisman \& } \\
\text { Borow. }\end{array}$ & Nemaliales & Galaxauraceae \\
\hline & $\begin{array}{c}\text { Tatagiba Beach } \\
\left(21^{\circ} 23^{\prime} 31.11^{\prime \prime S}, 40^{\circ} 59^{\prime} 9.64 " \mathrm{O}\right) \\
\text { Intertidal }\end{array}$ & Hypnea musciformis (Wulfen in Jacquin) J.V. Lamour. & Gigartinales & Cystocloniacae \\
\hline & & Corallina oficinallis & & \\
\hline & & Gracilaria cearensis & Gracilariales & Gracilariaceae \\
\hline & & Chondracanthus acicularis (Roth) Fredericq & Gigartinales & Gigartinaceae \\
\hline \multirow{8}{*}{ 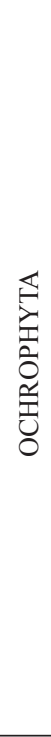 } & $\begin{array}{c}\text { Cabo Frio Island } \\
\left(23^{\circ} 0^{\prime} 10.02^{\prime \prime} \mathrm{S}, 42^{\circ} 0^{\prime} 24.43^{\prime \prime} \mathrm{O}\right) \\
\text { Infralittoral }\end{array}$ & Sargassum polyceratium Mont. & Fucales & Sargassaceae \\
\hline & $\begin{array}{c}\text { Forno Beach } \\
\left(22^{\circ} 44^{\prime} 31.70^{\prime \prime} \mathrm{S}, 41^{\circ} 52^{\prime} 35.97^{\prime \prime} \mathrm{O}\right) \\
\text { Infralittoral fringe }\end{array}$ & $\begin{array}{c}\text { Lobophora variegata (J.V. Lamour.) Womersley ex E.C. } \\
\text { Oliveira }\end{array}$ & Dictyotales & Dictyotaceae \\
\hline & $\begin{array}{c}\text { Forno Beach } \\
\left(22^{\circ} 44^{\prime} 31.70^{\prime \prime} \mathrm{S}, 41^{\circ} 52^{\prime} 35.97 " \mathrm{O}\right) \\
\text { Infralittoral }\end{array}$ & Stypopodium zonale (J.V. Lamour.) Papenf. & Dictyotales & Dictyotaceae \\
\hline & $\begin{array}{c}\text { Rasa Beach } \\
\left(22^{\circ} 44^{\prime} 3.15^{\prime \prime} \mathrm{S}, 41^{\circ} 57^{\prime} 30.15^{\prime \prime} \mathrm{O}\right) \\
\text { Intertidal }\end{array}$ & Padina gymnospora (Kütz.) Sond. & Dictyotales & Dictyotaceae \\
\hline & $\begin{array}{c}\text { Rasa Beach } \\
\left(22^{\circ} 44^{\prime} 3.15^{\prime \prime} \mathrm{S}, 41^{\circ} 57^{\prime} 30.15^{\prime \prime} \mathrm{O}\right)\end{array}$ & Dictyopteris delicatula J.V. Lamour. & Dictyotales & Dictyotaceae \\
\hline & & $\begin{array}{c}\text { Dictyota menstrualis (Hoyt) Schnetter, Hörning \& Weber- } \\
\text { Peukert }\end{array}$ & Dictyotales & Dictyotaceae \\
\hline & & Sargassum cymosum C. Agardh & Fucales & Sargassaceae \\
\hline & & Sargassum vulgare C. Agardh & Fucales & Sargassaceae \\
\hline \multirow{12}{*}{ 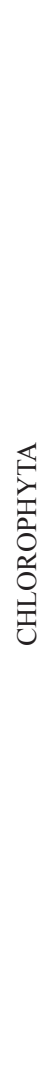 } & $\begin{array}{c}\text { Cavaleiros Beach } \\
\left(22^{\circ} 24^{\prime} 18.50^{\prime \prime} \mathrm{S}, 41^{\circ} 47^{\prime} 42.48^{\prime \prime} \mathrm{O}\right) \\
\text { Intertidal }\end{array}$ & Cladophora prolifera (Roth) Kütz. & Cladophorales & Cladophoraceae \\
\hline & $\begin{array}{c}\text { Forno Beach } \\
\left(22^{\circ} 44^{\prime} 31.70^{\prime \prime} \mathrm{S}, 41^{\circ} 52^{\prime} 35.97^{\prime \prime O}\right) \\
\text { Infralittoral fringe }\end{array}$ & Caulerpa racemosa (Forsskål) J. Agardh & Bryopsidales & Caulerpaceae \\
\hline & $\begin{array}{c}\text { Forno Beach } \\
\left(22^{\circ} 44^{\prime} 31.70^{\prime \prime S}, 41^{\circ} 52^{\prime} 35.97^{\prime \prime O}\right) \\
\text { Intertidal }\end{array}$ & Codium decorticatum (Woodw.) M. Howe & Bryopsidales & Codiaceae \\
\hline & $\begin{array}{c}\text { Francês Island } \\
\left(22^{\circ} 24^{\prime} 6.46 " \mathrm{~S}, 41^{\circ} 41^{\prime} 37.16^{\prime \prime} \mathrm{O}\right) \\
\text { Intertidal }\end{array}$ & Chaetomorpha antennina (Bory) Kütz. & Cladophorales & Cladophoraceae \\
\hline & $\begin{array}{c}\text { Francês Island } \\
\left(22^{\circ} 24^{\prime} 6.46^{\prime \prime} \mathrm{S}, 41^{\circ} 41^{\prime} 37.16^{\prime \prime} \mathrm{O}\right) \\
\text { Infralittoral }\end{array}$ & Codium spongiosum Harv. & Bryopsidales & Codiaceae \\
\hline & $\begin{array}{c}\text { Rasa Beach } \\
\left(22^{\circ} 44^{\prime} 3.15^{\prime \prime} \mathrm{S}, 41^{\circ} 57^{\prime} 30.15^{\prime \prime} \mathrm{O}\right) \\
\text { Intertidal }\end{array}$ & Bryopsis sp. & Bryopsidales & Bryopsidaceae \\
\hline & $\begin{array}{c}\text { Rasa Beach } \\
\left(22^{\circ} 44^{\prime} 3.15^{\prime \prime} \mathrm{S}, 41^{\circ} 57^{\prime} 30.15^{\prime \prime} \mathrm{O}\right) \\
\text { Intertidal }\end{array}$ & Ulva fasciata Delile & Ulvales & Ulvaceae \\
\hline & $\begin{array}{c}\text { Centro Beach } \\
\left(17^{\circ} 638.86^{\prime \prime} \text { S3910' } 54.95^{\prime \prime} \mathrm{W}\right) \\
\text { Intertidal }\end{array}$ & Avrainvillea elliotti A. Gepp \& E.S. Gepp & Bryopsidales & Udoteaceae \\
\hline & & Udotea flabellum (J. Ellis \& Solander) M. A. Howe & Bryopsidales & Udoteaceae \\
\hline & & Halimeda opuntia J.V. Lamouroux & Bryopsidales & Halimedaceae \\
\hline & & Halimeda tuna tuna (J. Ellis \& Solander) J.V. Lamouroux & Bryopsidales & Halimedaceae \\
\hline & & Penicillus capitatus Lamarck & Bryopsidales & Udoteaceae \\
\hline
\end{tabular}




\section{Cells and viruses}

Vero cells were grown in Eagle's minimum essential medium (MEM) supplemented with $2 \mathrm{mM}$ L-glutamine, $50 \mu \mathrm{g} / \mathrm{mL}$ gentamicin, $2.5 \mu \mathrm{g} / \mathrm{mL}$ fungizon, plus $10 \%$ of heat-inactivated fetal bovine serum (FBS) (Schmidt, 1979) and kept at $37{ }^{\circ} \mathrm{C}$ in an atmosphere of $5 \% \mathrm{CO}_{2}$. Acyclovir-resistant $\mathrm{HSV}-1$ and HSV-2 strains isolated from typical oral and genital lesions, respectively, were used. The isolates were typed by the polymerase chain reaction (PCR) using specific primers to identify HSV-1 and HSV-2 (Markoulatos et al., 2001) and evaluated with regard to sensibility to Acyclovir (De La Iglesia et al., 1998).

\section{Cytotoxicity}

The algal extracts were solubilized in dimethylsulfoxide (final concentration 1\%) and diluted in water to a concentration of $400 \mu \mathrm{g} / \mathrm{mL}$, sterilized by filtration through a Millipore membrane filter $(0.22$ $\mu \mathrm{m})$ and frozen at $-20{ }^{\circ} \mathrm{C}$ until use. The cytotoxicity assay was performed by incubating, in triplicate, Vero cell monolayers cultivated in 96-well microplates with two-fold serial dilutions of the extracts for 48 $\mathrm{h}$ at $37{ }^{\circ} \mathrm{C}$. Morphological alterations of the treated cells were observed in an inverted optical microscope (Leitz-Germany 633456), and the maximum non-toxic concentrations (MNTC) were determined (Walker et al., 1972). Cellular viability was further evaluated by the neutral red dye-uptake method (Neyndorff et al., 1990). The $50 \%$ cytotoxic concentration (CC50) was defined as the dilution that caused a reduction of $50 \%$ in the number of viable cells.

\section{Antiviral assays}

Anti-HSV activity was evaluated by reduction of the virus titers using TCID50 (50\% tissue culture infective dose) determinations. Vero cell monolayers cultivated in 96-well microplates were treated with the algal extracts at the MNTC. Immediately after, logarithmical dilutions of HSV-1 and HSV-2 suspensions were added to treated and untreated cell cultures and incubated in a $5 \% \mathrm{CO}_{2}$ atmosphere for $48 \mathrm{~h}$ at $37{ }^{\circ} \mathrm{C}$. Following incubation, the virus titers were calculated using the Reed \& Muench (1938) statistical method and expressed as TCID50 values. Results of the antiviral activity were expressed as Percentage of Inhibition (PI) (Nishimura et al., 1977) using antilogarithmic values of the TCID50 values as follows: PI=[1-(antilogarithm of the test value/antilogarithm of the control value)] 100 .

\section{Results}

A total of 36 macroalgae species from seven sites along the Brazilian coast were tested against acyclovir-resistant HSV-1 and HSV-2. Of these, sixteen species were Rhodophyta (44.4\%), eight species were Ochrophyta (22.2\%) and twelve species were Chlorophyta (33.3\%, Table 1). The results of the antiviral activity were expressed as PI. All results are reported in Table 2.

Out of all the crude extracts, 31 (86.1\%) showed some activity against HSV-1 (PI values ranging from 20.6 to $99.9 \%)$ and $20(55.5 \%)$ some activity against HSV-2 (PI values ranging from 20.6 to $96.0 \%$ ). Among the three Phyla, the Ochrophyta showed the highest percentages of active extracts against HSV-1, with $100 \%$ of the extracts exhibiting activity. The Chlorophyta and Rhodophyta represented 91.6 and $75.0 \%$, respectively of the active extracts. However, against HSV-2, Chlorophyta exhibited the highest percentage of active extracts (66.7\%), followed by Ochrophyta (62.5\%) and Rhodophyta (43.7\%). A strong anti-herpetic activity was considered for extracts with PI $>90 \%$. Eleven species (30.5\%), listed in Table 2, showed anti-HSV-1 activity with a PI superior to $90 \%$. Among these, $L$. dendroidea, S. zonale, S. cymosum, U. fasciata and $C$. decorticatu showed very high activities (97.5, 96.8, 98.2, 99.9 and $99.9 \%$ respectively). On the other hand, strong anti-HSV-2 activity (PI $>90 \%)$ was observed in only four species $(11.1 \%)$, i.e., S. zonale, S. cymosum, C. acicularis and $P$. capitatus, with $P$. capitatus being the most active $(96.0 \%)$. All algal extracts with strong anti-HSV activity (PI $>90 \%$ ) presented no toxicity to Vero cells (CC50>200 $\mu \mathrm{g} / \mathrm{mL}$ ), except the extract from Laurencia dendroidea (CC50 $48.2 \mu \mathrm{g} / \mathrm{mL}$ ).

All the crude extracts were analyzed by ${ }^{1} \mathrm{H}-\mathrm{NMR}$ and ${ }^{13} \mathrm{C}-\mathrm{NMR}$ spectroscopy. The major constituents of the most active extracts (L. dendroidea, S. zonale, S. cymosum, U. fasciata, C. decorticatum and $P$. capitatus) were identified. Characteristic signals for terpenoids were observed in the crude extracts from the algae L. dendroidea and $S$. zonale. Comparison of the spectroscopic data with previously reported data allowed the identification of the halogenated sesquiterpenes obtusol (1) and (-)-elatol (2) from $L$. dendroidea and the meroditerpenoid atomaric acid (3) from S. zonale (González et al., 1979; Wessels et al., 1999; Soares et al., 2003; Machado et al., 2011). The phenolic composition of the S. cymosum extract was suggested by the signals in the ${ }^{1} \mathrm{H}$ NMR spectrum at $\delta 6.54(\mathrm{bs}), 6.51(\mathrm{~d}, J=3.0 \mathrm{~Hz}), 6.48(\mathrm{~d}, J=3.0 \mathrm{~Hz})$ and 6.45 (bs), characteristic to two coupled aromatic protons meta to each other, and a group of the signals at $160.0-120.0 \mathrm{ppm}$ in the ${ }^{13} \mathrm{C}$ NMR spectra, characteristic of a phenolic moiety. The presence of triacylglycerols and fatty acids as the major components from the algae $U$. fasciata, $C$. decorticatum and $P$. capitatus is indicated by the strong ${ }^{1} \mathrm{H}$ NMR signals at $\delta 4.29 \mathrm{ppm}$ 
Table 2. Benthic marine macroalgae extract activities against acyclovir resistant Herpes simplex viruses (HSV-1-ACVr; HSV-2$\mathrm{ACVr}$ ). CC50 or $50 \%$ cytotoxic concentration is the concentration required to reduce the number of viable Vero cells by $50 \%$ after $48 \mathrm{~h}$ of incubation with the extracts. MNTC or maximum non-toxic concentration is the maximum concentration that did not cause morphologic alterations of the treated Vero cells. PI: percentage of inhibition.

\begin{tabular}{|c|c|c|c|c|}
\hline Species & $\operatorname{CC} 50(\mu \mathrm{g} / \mathrm{mL})$ & CMNT $(\mu \mathrm{g} / \mathrm{mL})$ & $\begin{array}{c}\text { PI (\%) } \\
\text { (HSV-1-ACVr) }\end{array}$ & $\begin{array}{c}\text { PI (\%) } \\
\text { (HSV-2-ACVr) }\end{array}$ \\
\hline \multicolumn{5}{|l|}{ Rodophyta } \\
\hline Corallina panizzoii & 175.8 & 100 & 68.4 & Zero \\
\hline Jania adhaerens & $>200$ & 50 & Zero & Zero \\
\hline Jania crassa & 173.5 & 100 & 43.8 & 43.8 \\
\hline Tricleocarpa cylindrica & $>200$ & 200 & 83.4 & Zero \\
\hline Bostrychia radicans & $>200$ & 200 & 86.5 & Zero \\
\hline Centroceras clavulatum & $>200$ & 200 & zero & Zero \\
\hline Laurencia dendroidea & 48.2 & 3.1 & 97.5 & 43.8 \\
\hline Osmundaria obtusiloba & $>200$ & 100 & 90 & Zero \\
\hline Spyrdia clavata & $>200$ & 200 & 85.9 & 20.6 \\
\hline Pterocladiella capillacea & $>200$ & 50 & 68.4 & Zero \\
\hline Hypnea musciformis & $>200$ & 100 & 57.3 & 74.9 \\
\hline Hypnea spinella & $>200$ & 200 & 92 & Zero \\
\hline Chondracanthus acicularis & $>200$ & 100 & 68.4 & 92.4 \\
\hline Gracilaria domingensis & $>200$ & 100 & Zero & 43.8 \\
\hline Cryptonemia seminervis & $>200$ & 100 & Zero & Zero \\
\hline Plocamium brasiliense & $>200$ & 200 & 43.8 & 77.6 \\
\hline \multicolumn{5}{|l|}{ Ochrophyta } \\
\hline Dictyopteris delicatula & $>200$ & 100 & 82.2 & 77.6 \\
\hline Dictyota menstrualis & 94.5 & 12.5 & 20.6 & Zero \\
\hline Lobophora variegata & 33.8 & 6.2 & 92 & Zero \\
\hline Padina gymnospora & $>200$ & 100 & 85.9 & 43.8 \\
\hline Stypopodium zonale & $>200$ & 50 & 96.8 & 95.8 \\
\hline Sargassum cymosum & 124.4 & 50 & 98.2 & 90 \\
\hline Sargassum polyceratium & 194.5 & 100 & 86.8 & Zero \\
\hline Sargassum vulgare & 128.8 & 50 & 76 & 39.7 \\
\hline \multicolumn{5}{|l|}{ Chlorophyta } \\
\hline Ulva fasciata & $>200$ & 200 & 99.9 & Zero \\
\hline Chaetomorpha antennina & 85.8 & 100 & 55.3 & 85.9 \\
\hline Cladophora prolifera & $>200$ & 50 & 90 & Zero \\
\hline Bryopsis sp. & $>200$ & 200 & 82.2 & 87.4 \\
\hline Codium decorticatum & $>200$ & 200 & 99.9 & Zero \\
\hline Codium spongiosum & $>200$ & 50 & 55.3 & 55.3 \\
\hline Caulerpa racemosa & $>200$ & 50 & 57.3 & Zero \\
\hline Avrainvillea elliottii & $>200$ & 125 & Zero & 60.2 \\
\hline Udotea flabellum & $>200$ & 125 & 90 & 75 \\
\hline Halimeda opuntia & $>200$ & 62.5 & 73.1 & 68.4 \\
\hline Halimeda tuna & $>200$ & 250 & 84.1 & 82.2 \\
\hline Penicillus capitatus & $>200$ & 250 & 93.0 & 96 \\
\hline
\end{tabular}


(dd, $J=7.4 ; 14.6 \mathrm{~Hz}), 4.16 \mathrm{ppm}(\mathrm{dd}, J=6.0 ; 12.4 \mathrm{~Hz}$ ) and $5.36 \mathrm{ppm}(\mathrm{m})$, characteristic of triacylglycerols. The strong signal from the terminal methyl groups of the fatty acid esters were clearly observed at $\delta 0.88 \mathrm{ppm}$ (t, $J=7.2 \mathrm{~Hz}$ ). The signals at $\delta 2.31 \mathrm{ppm}(\mathrm{t}, J=7.4 \mathrm{~Hz}$ ) and $1.60 \mathrm{ppm}(\mathrm{m})$ correspond to the methylene protons $\alpha$ - and $\beta$ - to the carbonyl groups, respectively. Peaks at $\delta 2.80 \mathrm{ppm}$ and $2.02 \mathrm{ppm}$ are attributed to methylene protons adjacent to double bonds. A strong peak for the internal methylene groups of the long chain of the fatty acid esters was observed at $\delta 1.26 \mathrm{ppm}$ (bs). The ${ }^{13} \mathrm{C}$ NMR spectra of the extracts showed resonances of fatty acid ester carboxyl groups and the signals at 60 $70 \mathrm{ppm}$ indicated the presence of the glycerol moiety. All of the NMR data are shown in Table 3.

\section{Discussion}

Several molecules extracted from marine algae possess a broad spectrum of antiviral activity. Chemical classes for these compounds include sterols, terpenes, acetogenins, polyssacharides, fatty acids and polyphenols (Pereira et al., 2004a; Maschek \& Baker, 2008; Hidari et al., 2008). In this study, we investigated the anti-herpetic activity against acyclovir-resistant HSV-1 and HSV-2 of lipophilic extracts from 36 Brazilian seaweeds. Of all the crude extracts, $31(86.1 \%)$ showed some activity against HSV-1 and $20(55.5 \%)$ some activity against HSV-2. The most active anti-HSV extracts were obtained from the species $L$. dendroidea, U. fasciata, C. decorticatum, $S$. zonale, S. cymosum, C. acicularis and P. capitatus.<smiles>C=C1C[C@@H](O)[C@H](Br)C(C)(C)[C@]12CCC(C)(Br)C(Cl)C2</smiles>

1<smiles>C=C1C[C@H](O)C(Br)C(C)(C)[C@]12CCC(C)C(Cl)C2</smiles>

2<smiles>COc1cc(C)c(O)c(C[C@]2(C)[C@H](C)CC[C@H]3[C@@H](CCC(=O)O)C(=C(C)C)CC[C@H]32)c1</smiles>

3

Table 3. ${ }^{1} \mathrm{H}-\left(\mathrm{CDCl}_{3}, 300 \mathrm{MHz}\right)$ and ${ }^{13} \mathrm{C}-\mathrm{NMR}\left(\mathrm{CDCl}_{3}, 75.5 \mathrm{MHz}\right)$ data for the major components of the crude extracts from Laurencia dendroidea, Stypopodium zonale, Ulva fasciata, Codium decorticatum, Penicillus capitatus and Sargassum cymosum.

\begin{tabular}{|c|c|c|c|c|}
\hline Compound & Assignment & ${ }^{1} \mathrm{H}(\mathrm{ppm})$ & Multiplicity: $J(\mathrm{~Hz})$ & ${ }^{13} \mathrm{C}(\mathrm{ppm})$ \\
\hline \multicolumn{5}{|c|}{ L. dendroidea } \\
\hline \multirow[t]{17}{*}{ obtusol (1) } & $\mathrm{CH}-1$ & 1.74 & $m$ & 25.5 \\
\hline & $\mathrm{CH}-2$ & 2.30 & $d m(12.6)$ & 40.5 \\
\hline & $\mathrm{CH}-3$ & - & - & 67.6 \\
\hline & $\mathrm{CH}-4$ & 4.70 & $d d(10.8$ and 2.9$)$ & 68.0 \\
\hline & $\mathrm{CH}-5$ & & & 37.1 \\
\hline & CH-6 & - & & 50.0 \\
\hline & $\mathrm{CH}-7$ & - & & 141.8 \\
\hline & $\mathrm{CH}-8$ & 2.62 & $d(14.0)$ & 38.5 \\
\hline & & 2.50 & $d(14.2)$ & \\
\hline & CH-9 & 4.10 & $b s$ & 72.0 \\
\hline & $\mathrm{CH}-10$ & 4.48 & $d(3.0)$ & 70.1 \\
\hline & $\mathrm{CH}-11$ & - & & 44.0 \\
\hline & $\mathrm{CH}-12$ & 1.08 & $s$ & 24.0 \\
\hline & $\mathrm{CH}-13$ & 1.08 & $s$ & 20.8 \\
\hline & $\mathrm{CH}-14$ & 5.39 & $b s$ & 117.8 \\
\hline & & 5.05 & $b s$ & \\
\hline & $\mathrm{CH}-15$ & 1.83 & $s$ & 23.6 \\
\hline \multirow[t]{7}{*}{ (-)-elatol (2) } & $\mathrm{CH}-1$ & 2.58 & $d l(17.5)$ & 25.5 \\
\hline & & 2.36 & $d l(17.5)$ & \\
\hline & $\mathrm{CH}-2$ & - & - & 128.0 \\
\hline & $\mathrm{CH}-3$ & - & - & 124.1 \\
\hline & $\mathrm{CH}-4$ & 1.98 & $m$ & 29.5 \\
\hline & $\mathrm{CH}-5$ & 1.63 & $m$ & 25.6 \\
\hline & CH-6 & - & - & 49.4 \\
\hline
\end{tabular}




\begin{tabular}{|c|c|c|c|c|}
\hline & $\mathrm{CH}-7$ & - & & 140.8 \\
\hline & $\mathrm{CH}-8$ & 2.62 & $d d(14.4$ and 2.71$)$ & 33.5 \\
\hline & & 2.49 & $d m(14.5)$ & \\
\hline & $\mathrm{CH}-9$ & 4.14 & $d(2.9)$ & 72.2 \\
\hline & $\mathrm{CH}-10$ & 4.60 & $d(2.9)$ & 70.4 \\
\hline & $\mathrm{CH}-11$ & - & - & 43.2 \\
\hline & $\mathrm{CH}-12$ & 1.05 & $b s$ & 20.7 \\
\hline & $\mathrm{CH}-13$ & 1.06 & $b s$ & 24.2 \\
\hline & $\mathrm{CH}-14$ & 5.12 & $b s$ & 115.2 \\
\hline & & 4.80 & $s$ & \\
\hline & $\mathrm{CH}-15$ & 1.70 & $s$ & 19.4 \\
\hline & & S. zonc & & \\
\hline atomaric acid (3) & $\mathrm{CH}-1$ & 2.84 & $d(13.8)$ & 34.7 \\
\hline & & 2.25 & $d(14.3)$ & \\
\hline & $\mathrm{CH}-2$ & - & & 40.0 \\
\hline & $\mathrm{CH}-3$ & 1.73 & $m$ & 35.1 \\
\hline & $\mathrm{CH}-4$ & 1.26 & $d(14.4)$ & 24.9 \\
\hline & $\mathrm{CH}-5$ & 1.49 & $m$ & 36.1 \\
\hline & $\mathrm{CH}-6$ & - & & 38.5 \\
\hline & $\mathrm{CH}-7$ & 1.38 & $d d(12.0$ and 6.0$)$ & 41.8 \\
\hline & $\mathrm{CH}-8$ & 1.74 & $m$ & 22.2 \\
\hline & CH-9 & 2.39 & $m$ & 23.3 \\
\hline & $\mathrm{CH}-10$ & - & $m$ & 122.3 \\
\hline & $\mathrm{CH}-12$ & 1.81 & $m$ & \\
\hline & $\mathrm{CH}-12$ & 1.81 & $m$ & \\
\hline & $\mathrm{CH}-13$ & 2.26 & $m$ & 32.7 \\
\hline & $\mathrm{CH}-14$ & - & & 174.9 \\
\hline & $\mathrm{CH}-15$ & - & & 133.3 \\
\hline & $\mathrm{CH}-16$ & 1.68 & $s$ & 20.4 \\
\hline & $\mathrm{CH}-17$ & 1.66 & $s$ & 20.6 \\
\hline & $\mathrm{CH}-18$ & 1.02 & $s$ & 17.8 \\
\hline & $\mathrm{CH}-19$ & 0.93 & $s$ & 20.4 \\
\hline & $\mathrm{CH}-20$ & 1.15 & $d(6.9)$ & 15.5 \\
\hline & $\mathrm{CH}-1$ & - & & 147.5 \\
\hline & $\mathrm{CH}-2^{\prime}$ & - & & 128.3 \\
\hline & $\mathrm{CH}-3$ & 6.69 & $d(3.0)$ & 113.6 \\
\hline & $\mathrm{CH}-4$ & - & & 151.7 \\
\hline & $\mathrm{CH}-5$ & 6.54 & $d(2.7)$ & 112.9 \\
\hline & $\mathrm{CH}-6$, & - & & 125.5 \\
\hline & $\mathrm{CH}-7^{\prime}$ & 2.22 & $s$ & 17.5 \\
\hline & $\mathrm{CH}-8$ & 3.73 & $s$ & 55.0 \\
\hline \multicolumn{5}{|c|}{ U. fasciata, C. decorticatum and P. capitatus } \\
\hline \multirow[t]{3}{*}{ triacylglycerols } & $\mathrm{CH} s n-2$ & 5.26 & $\mathrm{~m}$ & 69.0 \\
\hline & $\mathrm{CH}_{2} s n-1,3$ & 4.29 & $d d,(7.4$ and 14.6$)$ & 62.0 \\
\hline & & 4.16 & $d d(6.0$ and 12.4$)$ & \\
\hline \multirow[t]{4}{*}{ fatty acids } & $\mathrm{COO}$ & - & - & 172.8 \\
\hline & $\omega-\mathrm{CH}_{3}$ & 0.88 & $t(7.2)$ & 14.2 \\
\hline & $\alpha-\mathrm{CH}_{2}$ & 2.31 & $t(7.4)$ & \\
\hline & $\beta-\mathrm{CH}_{2}$ & 1.60 & $m$ & \\
\hline
\end{tabular}




\begin{tabular}{cccc}
\hline & $\mathrm{CH}_{2}-\mathrm{C}=$ & 2.80 & $m$ \\
& & 2.02 & $m$ \\
& $\left(\mathrm{CH}_{2}\right)_{\mathrm{n}}-\mathrm{CH}_{3}$ & 1.26 & $b s$ \\
\hline phenolic compounds & & S. cymosum & \\
& 6.54 & $b s$ & \\
6.51 & $d(3.0)$ & \\
6.48 & $d(3.0)$ & $b s$ \\
\hline
\end{tabular}

Red algae of the genus Laurencia are found in tropical and subtropical regions throughout the world and are an extremely rich source of secondary metabolites with diverse structural features, mainly halogenated terpenes and C15-acetogenins, with a broad spectrum of biological activity (Machado et al., 2010). The halogenated sesquiterpenes obtusol (1) and (-)-elatol (2) were identified as the major compounds of L. dendroidea.

The green algae $U$. fasciata, $C$. decorticatum and $P$. capitatus showed high activity against HSV-1. These genera had high concentrations of polysaccharides and fatty acids (Pope et al., 1996). These compounds may be responsible for the observed activity. Fatty acid-treated cells are resistant to infection by a variety of lipid-enveloped viruses, including herpes viruses (Pope et al., 1998). The chemical profiles of the crude extracts, obtained by the use of ${ }^{1} \mathrm{H}-\mathrm{NMR}$ and ${ }^{13} \mathrm{C}-\mathrm{NMR}$ spectroscopy, showed the presence of triacylglycerols and a mixture of fatty acids as the major components in these extracts.

The brown algae of the genera Stypopodium (Dictyotales) and Sargassum (Fucales) are abundantly found along the Brazilian coast. Both genera are known to produce meroditerpenes (mixed biogenesis diterpenes). Other metabolites of structural classes such as glycerides (Tang et al., 2002a), steroids (Tang et al., 2002b), dipeptides (Liu et al., 2009) and flavonoids (Liu et al., 2009) were isolated from the genus Sargassum. The species $S$. cymosum together with $S$. zonale were the only species that were highly active against both the viruses HSV-1 and HSV-2. The meroditerpenoid atomaric acid (3) was identified as the major secondary metabolite in the $\mathrm{S}$. zonale extract. Meroditerpenes from S. zonale are known to have diverse biological activities (Wessels et al., 1999; Sabry et al., 2005), including anti-HSV-1 activity (Soares et al., 2007). The ${ }^{1} \mathrm{H}-\mathrm{NMR}$ spectroscopic data of the crude extract of S. cymosum showed signals characteristic of phenolic compounds as the major constituents. Phenolic compounds have received considerable attention because of their therapeutic effects and their favorable antiviral activity (Quideau et al., 2004; Likhitwitayawuid, 2005; Tareq et al., 2007).

Although it is not possible to determine whether only one or a combination of several molecules are responsible for the observed anti-HSV-1 and antiHSV-2 activity of the extracts, the presence of terpenes, fatty acids and phenolic compounds is consistent with the observed anti-herpetic activity since these types of metabolites, isolated from marine and terrestrial sources, have already been shown to have anti-herpetic activity (Khan et al., 2005; Hayashi et al., 2008).

The results of the present study indicate that different crude extracts from marine algae exhibit high anti-herpetic activity. The present findings provide a basis for further experiments on the identification and characterization of specific compounds with high antiherpetic activities.

\section{Acknowledgment}

The authors thank Soluza dos Santos Gonçalves for technical assistance. This study was supported by the Conselho Nacional de Desenvolvimento Científico e Tecnológico (CNPq), the Coordenação de Aperfeiçoamento de Pessoal de Nível Superior (CAPES), FINEP (Number of process: 3175/06) and Fundação Carlos Chagas de Amparo à Pesquisa do Estado do Rio de Janeiro (FAPERJ), Brazil. We thank Heitor Monteiro Duarte and Tatiana Konno for valuable comments.

\section{References}

Amsler CD, Fairhead VA 2005. Defensive and sensory chemical ecology of brown algae. Adv Bot Res 43: 1-91.

Barbier P, Guise S, Huitorel P, Amade P, Pesando D, Briand C, Peyrot V 2001. Caulerpenyne from Caulerpa taxifolia has an antiproliferative activity on tumor cell line SK$\mathrm{N}-\mathrm{SH}$ and modifies the microtubule network. Life Sci 70: 415-29.

Beach K, Walters L, Borgeas H, Smith C, Coyer J, Vroom P 2003. The impact of Dictyota spp. on Halimeda populations of Conch Reef, Florida Keys. J Exp Mar Biol Ecol 297: 141-159.

Brown TJ, McCrary M, Tyring SK 2002. Antiviral agents: Nonantiviral drugs. J Am Acad Dermatol 47: 581-599.

Cirne-Santos CC, Souza TML, Teixeira VL, Fontes CFL, Rebello MA, Castello-Branco LRR, Abreu CM, 
Tanuri A, Frugulhetti ICPP, Bou-Habib DC 2008. The dolabellane diterpene dolabelladienetriol is a typical noncompetitive inhibitor of HIV-1 reverse transcriptase enzyme. Antivir Res 77: 64-71.

Da Gama BAP, Carvalho AGV, Weidner K, Soares AR, Coutinho R, Fleury BG 2008. Antifouling activity of natural products from Brazilian seaweeds. Bot Mar 51: 191-201.

Davyt D, Fernandez R, Suescun L, Mombru AW, Saldan J, Domínguez L, Coll J, Fujii MT, Manta E 2001. New sesquiterpene derivatives from the red alga Laurencia scoparia. Isolation, structure determination, and anthelmintic activity. $J$ Nat Prod 64: 1552-1555.

De Clercq E 2005. Antiviral drug discovery and development: Where chemistry meets with biomedicine. Antivir Res 67: 56-75.

De La Iglesia P, Melón S, López B, Rodríguez M, Blanco MI, Mellado P, De Oña M 1998. Rapid screening tests for determining in vitro susceptibility of Herpes simplex virus clinical isolates. J Clin Microbiol 36: 2389-2391.

De Oliveira ALL, Felicio R, Costa-Lotufo LV, Moraes MO, Pessoa CO, Young MCM, Yokoya NC, Debonsi HM 2008. Antitumor and antifungal activities from red algae Bostrychia radicans and $B$. tenella (Rhodophyta). Planta Med 74: 977-977.

Ghosh P, Adhikari V, Ghosal PK, Pujol CA, Carlucci MJ, Damonte EB, Ray B 2004. In vitro anti-herpetic activity of sulfated polysaccharide fractions from Caulerpa racemosa. Phytochemistry 65: 3151-3157.

Gomez I, Rodriguez EP, Vinegla B, Figueroa FL, Karsten U 1998. Effects of solar radiation on photosynthesis, UV-absorbing compounds and enzyme activities of the green alga Dasycadus verrnicularis from southern Spain. J Photoch Photobio B 47: 46-57.

González AG, Martín JD, Martín VS, Norte M 1979. Carbon ${ }^{13} \mathrm{NMR}$ application to Laurencia polyhalogenated sesquiterpenes. Tetrahedron Lett 29: 2719-2722.

Hayashi K, Nakano T, Hashimoto M, Kanekiyo K, Hayashi $\mathrm{T}$ 2008. Defensive effects of a fucoidan from brown alga Undaria pinnatifida against herpes simplex virus infection. Int Immunopharmacol 8: 109-116.

Hidari KIPJ, Takahashia N, Arihara M, Nagaoka M, Morita K, Suzuki T 2008. Structure and anti-dengue virus activity of sulfated polysaccharide from a marine alga. Biochem Bioph Res Co 376: 91-95.

Jerome KR 2005. The road to new antiviral therapies. Clin Appl Immunol Rev 5: 65-76.

Khan MTH, Ather A, Thompsonc KD, Gambari R 2005. Extracts and molecules from medicinal plants against herpes simplex viruses. Antivir Res 67: 107-119.

Laillea M, Geraldb F, Debitus C 1998. In vitro antiviral activity on dengue virus of marine natural products. Cell Mol Life Sci 54: 167-170.

Likhitwitayawuid K, Supudompol B, Sritularak B, Lipipun V, Rapp K, Schinazi RF 2005. Phenolics with anti-HSV and anti-HIV activities from Artocarpus gomezianus, Mallotus pallidus and Triphasia trifolia. Pharm Biol 43: 651-657.

Lima LMD, Alor R, Uriostegui R, Murray SN, Pereira RC 2008. Within-plant variation in palatability and chemical defenses in the green seaweed Avrainvillea elliottii. Bot Mar 51: 21-25.

Liu X, Wang C-Y, Shao C-L, Wei Y-X, Wang B-G, Sun L-L, Zheng C-J, Guan H-S 2009. Chemical constituents from Sargassum pallidum (Turn.) C. Agardh. Biochem Syst Ecol 37: 127-129.

Machado FLS, Pacienza-Lima W, Rossi-Bergman B, Gestinari LM, Fujii M, de Paula JC, Costa SS, Lopes NP, Kaiser CR, Soares AR 2011. Antileishmanial sesquiterpenes from the Brazilian red alga Laurencia dendroidea. Planta Med 77: 733-735.

Machado FLS, Kaiser CR, Costa SS, Gestinari LM, Soares AR 2010. Atividade biológica de metabólitos secundários de algas do gênero Laurencia. Rev Bras Farmacogn 20: 441-452.

Markoulatos P, Georgopoulou A, Siafakas N, Plakokefalos E, Tzanakaki G, Kourea-Kremastinou J 2001. Laboratory diagnosis of common herpesvirus infections of the central nervous system by a multiplex PCR assay. J Clin Microbiol 39: 4426-4432.

Maschek JA, Baker BJ 2008. The chemistry of algal secondary metabolism. In: Amsler CD (ed.). Algal chemical ecology. Berlin Heidelberg: Springer-Verlag, p. 1-20.

Nahas R, Abatis D, Anagnostopoulou MA, Kefalas P, Vagias C, Roussis V 2007. Radical-scavenging activity of Aegean Sea marine algae. Food Chem 102: 577-581.

NeyndorffHC, Bartel DL, Tufaro F, Levy JG 1990. Development of a model to demonstrate photosensitizer-mediated viral inactivation in blood. Transfusion 30: 485-490.

Nishimura T, Toku K, Fukuyasu H 1977. Antiviral compounds. XII. Antiviral activity of aminohydrazones of alkoxyphenil substituted carbonyl compounds against influenza virus in eggs and mice. Kitasato Arch Exp Med 50: 39-46.

Paul VJ, Ritson-Williams R 2008. Marine chemical ecology. Nat Prod Rep 25: 662-695.

Pereira HS, Leão-Ferreira LR, Moussatché N, Teixeira VL, Cavalcanti DN, Costa LJ, Diaz R, Frugulhetti ICPP 2004a. Antiviral activity of diterpenes isolated from the Brazilian marine alga Dictyota menstrualis against human immunodeficiency virus type 1 (HIV-1). Antivir Res 64: 69-76.

Pereira RC, Soares AR, Teixeira VL, Villaça R, Da Gama BAP 2004b. Variation in chemical defenses against herbivory in southwestern Atlantic Stypopodium zonale (Phaeophyta). Bot Mar 47: 202-208.

Pope LE, Marcelletti JF, Katz LR, Lin JY, Katz DH, Parish ML, Spear PG 1998. The anti-herpes simplex virus activity of $n$-docosanol includes inhibition of the viral entry process. Antivir Res 40: 85-94. 
Pope LE, Marcelletti JF, Katz LR, Katz DH 1996. Anti-herpes simplex virus activity of $n$-docosanol correlates with intracellular metabolic conversion of the drug. J Lipid Res 37: 2167-2178.

Quideau S, Varadinova T, Karagiozova D, Jourdes M, Pardon P, Baudry C, Genova P, Diakov T, Petrova R 2004. Main structural and stereochemical aspects of the antiherpetic activity of nonahydroxyterphenoylcontaining $C$-glycosidic ellagitannins. Chem Biodivers 1: 247-258.

Reed LJ, Muench H 1938. A simple method of estimating fifty percents endpoints. Am J Hyg 27: 493-497.

Sabry OMM, Andrews S, McPhail KL, Goeger DE, Yokochi A, LePage KT, Murray TF, Gerwick WH 2005. Neurotoxic meroditerpenoids from the tropical marine brown alga Stypopodium flabelliforme. J Nat Prod 68: 1022-1030.

Santos MGM, Lagrota MHC, Miranda MMFS, YoneshigueValentin Y, Wigg MD 1999. A screening for the antiviral effect of Brazilian marine alga extracts against acyclovir-resistant herpes simplex virus type 1. Bot Mar 42: 227-230.

Schmidt NJ 1979. Cell culture techniques for diagnostic virology. In: Lennette EH, Schmidt NJ (eds.). Diagnostic procedures for viral and rickettsial infections. New York: American Publications Health Associations Inc., p. $65-139$.

Soares AR, Abrantes JL, Souza TML, Fontes CFL, Pereira RC, Frugulhetti ICPP, Teixeira VL 2007. Antiviral effect of meroditerpenes isolated from Stypopodium zonale (Dictyotaceae) against human immunodeficiency virus type 1 (HIV-1) and Herpes simplex virus (HSV-1). Planta Med 73: 1221-1224.

Soares AR, Teixeira VL, Pereira RC, Villaça R 2003. Variation on diterpene production by the Brazilian alga Stypopodium zonale (Dictyotales, Phaeophyta). Biochem Syst Ecol 31: 1347-1350.
Tang H, Yi Y, Yao X, Zhou D, Lu T, Jiang Y 2002a. Studies on bioactive steroid constituents from Sargassum carpophyllum. Chin Pharm J 37: 262-265.

Tang H, Yi Y, Yao X, Zhang S, Zou Z, Li L 2002b. Glycerides from marine brown algae Sargassum carpophyllum. Chin J Mar Drugs 21: 5-9.

Tareq M, Khan H, Ather A 2007. Potentials of phenolic molecules of natural origin and their derivatives as antiHIV agents. Biotech Ann Rev 13: 223-264.

Thiers B [continuously updated]. Index Herbariorum: A global directory of public herbaria and associated staff. New York botanical garden's virtual herbarium. http:// sweetgum.nybg.org/ih/.

Vairappan CS 2003. Potent antibacterial activity of halogenated metabolites from malaysian red algae Laurencia majuscula (Rhodomelaceae, Ceramiales). Biomol Eng 20: 255-259.

Walker WE, Waisbren BA, Martins RR, Batayas GE 1972. A method for determining sensitivities of antiviral drugs in vitro for possible use as clinical consultation. Appl Microbiol 23: 232-235.

Wessels M, König GM, Wright AD 1999. A new tyrosine kinase inhibitor from the marine brown alga Stypopodium zonale. J Nat Prod 62: 927-930.

\section{*Correspondence}

Angélica R. Soares

Grupo de Produtos Naturais de Organismos Aquáticos, Núcleo em Ecologia e Desenvolvimento Sócio-Ambiental de Macaé, Universidade Federal do Rio de Janeiro

Rua Rotary Club, s/n, São José do Barreto, Post Office Box 119331, 27910-970 Macaé-RJ, Brazil.

angelica@iq.ufrj.br

Tel.: +55-22-27593431

Fax: $+55-22-27599313$ 